\title{
THE LHCB TRACKING SYSTEM AND ITS PERFORMANCE
}

\author{
J. NARDULLI \\ On Behalf of the LHCb Collaboration \\ NIKHEF, \\ Kruislaan 409, \\ 1098 SJ Amsterdam, Netherlands \\ E-mail: jnardull@nikhef.nl
}

\begin{abstract}
$\mathrm{LHCb}$ is a next-generation forward spectrometer for CP violation measurements, using the Large Hadron Collider at CERN. In order to achieve its goals a high overall track reconstruction performance is needed. The LHCb tracking system comprises three main sub-systems: the vertex locator, the trigger tracker and the downstream tracking stations.
\end{abstract}

\section{Introduction}

LHCb aims to study $C P$ violation and rare $B$-meson decays with high precision, using the Large Hadron Collider (LHC), where all $B$-meson families are produced in $14 \mathrm{TeV} \mathrm{pp}$ collisions. In these events the $b \bar{b}$ pairs are typically produced in the same forward (or backward) direction. The $\mathrm{LHCb}$ detector is a single-arm spectrometer with a forward coverage from $10 \mathrm{mrad}$ to $300 \mathrm{mrad}$ in the horizontal plane (i.e., the bending plane of the magnet). The acceptance lies between 10-250 mrad in the vertical plane (non-bending plane). The detector layout in the bending plane is shown in Figure 1.

\section{Tracking System}

The LHCb Tracking system can be divided into three sub-systems.

- The VErtex LOcator (VELO) covers the area around the interaction point and is made of silicon sensors.

- The Trigger Tracker (TT), also built with silicon sensors, is downstream the interaction point, but in front of the large dipole mag- 


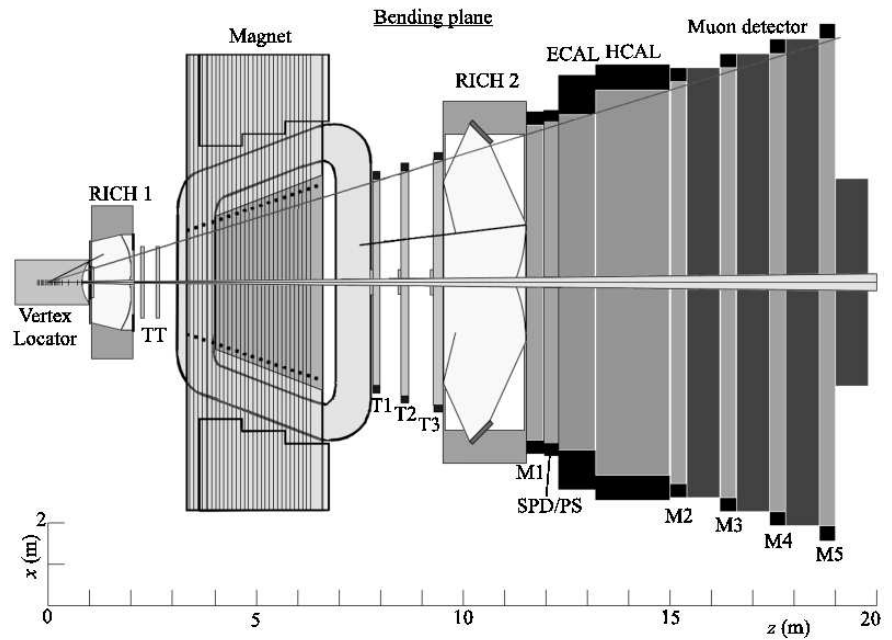

Figure 1. The LHCb setup with the different sub-detectors shown in the horizontal plane - also referred to as the bending plane of the magnet.

net.

- The Tracker Stations behind the magnet are divided in two parts: Inner Tracker (IT) and Outer Tracker (OT). The IT consists of silicon strip detectors, while the OT is made from straw tube drift cells.

\subsection{The Vertex Locator}

The Vertex Locator (VELO) $)^{1},{ }^{2}$ is made of 21 stations, placed along and perpendicular to the beam axis. Two different types of sensors are used: one measures the $r$ coordinate and the other measures the $\phi$ coordinate. The half-disk sensors, shown in Figure 2, are arranged in pairs of $r$ and $\phi$ sensors and mounted back-to-back.

The $r-\phi$ geometry is used for having a projection in the $r-z$ plane and for fast 2D-tracking in the trigger. The VELO has two main roles: it has to provide precise coordinate measurements to allow determination of primary and secondary vertices, and it is the main tracking detector before the magnet. 

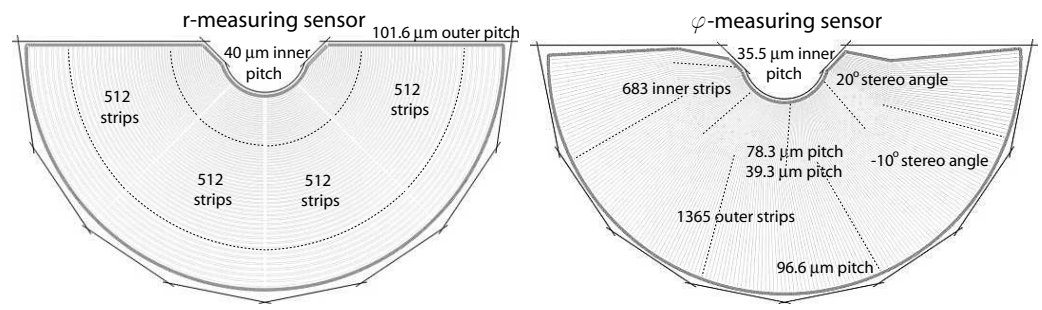

Figure 2. Layout of the VELO $r$ and $\phi$ measuring sensors.

\subsection{The Trigger Tracker}

The Trigger Tracker (TT) is located downstream the first RICH (Ring Imaging CHerenkov) detector and just in front of the magnet. It consists of two stations separated by a distance of $27 \mathrm{~cm}$. Every station consists of two layers, for a total of four layers, with angles between the $y$ and the $z$ axes in the following configuration: $0^{\circ},+5^{\circ},-5^{\circ}, 0^{\circ}$.

The Trigger Tracker main function is to provide momentum information to the trigger system; it is therefore mostly used for the so-called VELO-TT Tracking and to get a first estimate of the momentum, as it feels the fringe field of the magnet (see Figure 3).

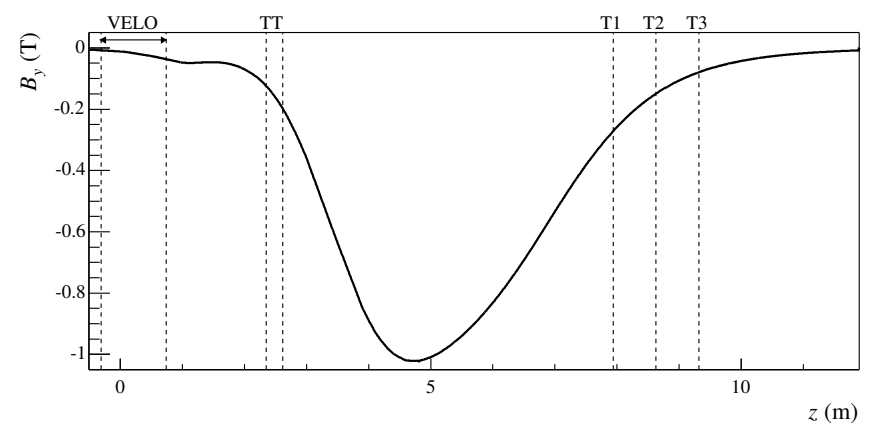

Figure 3. The main component of the magnetic field strength $\left(B_{y}\right)$ along the $z$ axis.

The silicon sensors are $500 \mu m$ thick and the strip pitch is $183 \mu m$. The maximum occupancy is approximately $2 \%$. 


\subsection{Tracker Stations}

The Tracker Stations located after the magnet cover an area of about $6 \times 5$ $m^{2}$. Over this area there is a large difference in the particle flux. In order to deal with this, the innermost part is covered with silicon strips (IT), while the outer part is covered with straw tube drift cells (OT). There are in total three Tracker Stations with four layers each. For the four different layers the stereo angles between the $y$ and $z$ axis are $0^{\circ},+5^{\circ},-5^{\circ}, 0^{\circ}$.

\subsubsection{The Inner Tracker}

The Inner Tracker ${ }^{3}$ covers approximately $2 \%$ of the area of the Tracker Stations, which corresponds to about $20 \%$ of the particle flux. The detectors are divided in boxes with 320 or $410 \mu m$ thick silicon. The strip pitch is $198 \mu \mathrm{m}$. The maximum occupancy is approximately $2 \%$.

\subsubsection{The Outer Tracker}

In the Tracker Stations, the Outer Tracker $(\mathrm{OT})^{4}$ covers the large region outside the acceptance of the Inner Tracker. The Outer Tracker consists of straw tube detectors with a diameter of $5 \mathrm{~mm}$. The gas mixture in use is $\operatorname{Ar}(70 \%) / \mathrm{CO}_{2}(30 \%)$. The signal collection is within $3 \mathrm{LHC}$ beam crossings $(75 \mathrm{~ns})$. Each of the three stations consists of four double layers of straws for a total of approximately 53.000 channels. The average occupancy is approximately $4.5 \%$, while the maximum occupancy is approximately $9 \%$.

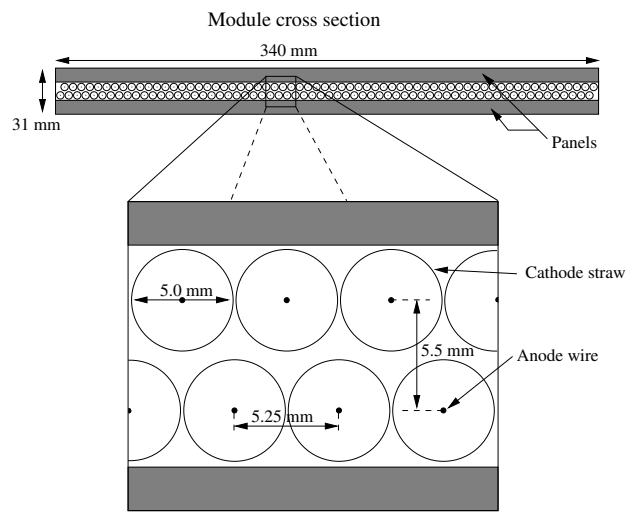

Figure 4. Cross section of an OT module (128 straws). A small region containing a few straws is magnified. 
The wires in the $4.8 \mathrm{~m}$ modules are split in the middle and the read-out is both at the top and at the bottom. A cross section of an Outer Tracker module is shown in Figure 4. The spatial resolution obtained in beam tests ${ }^{5}$ with this gas is approximately $200 \mu m$.

\section{Tracking Strategy and Performance}

$\mathrm{LHCb}$ is a challenging environment for tracking. Typically a particle sees $40 \%$ of a radiation length up to RICH2. A robust tracking strategy is therefore needed. Track finding starts by reconstructing tracks in the VELO. These tracks are extrapolated through the detector and matched to hits in the T Stations to form "long tracks". The unused hits are then considered to reconstruct the $\mathrm{T}$ seeds in the Tracker Stations. These $\mathrm{T}$ seeds are also matched to tracks in the VELO which did not get $\mathrm{T}$ hits added at the beginning. Upstream tracks are found with hits in the VELO and in the $\mathrm{TT}$, while tracks which have hits in the $\mathrm{TT}$ and in the $\mathrm{T}$ stations are called downstream tracks. The various track types are sketched in Figure 5.

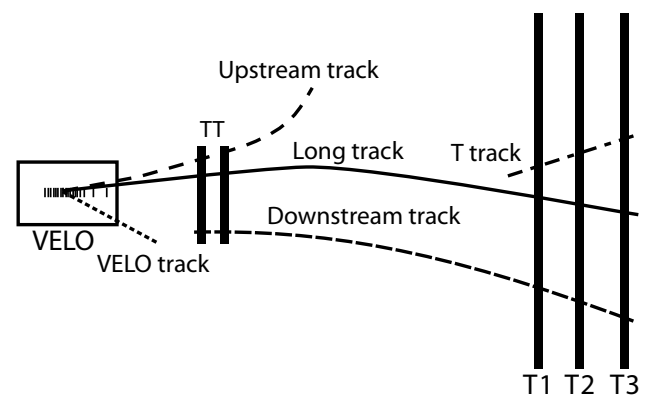

Figure 5. Sketch of the five different track types in the LHCb tracking system.

On average an event contains 72 reconstructed tracks, which can be divided into 26 long, 11 upstream, 4 downstream, $5 \mathrm{~T}$ and 26 VELO tracks. In Figure 6 the efficiency for finding long tracks and the ghost rate are shown as a function of the momentum.

The overall track reconstruction performance together with the excellent momentum resolution $(\Delta p / p=0.38 \%)$ leads to a typical resolution on the $B_{s}^{0}$-meson mass of $14 \mathrm{MeV} / \mathrm{c}^{2}$. The momentum resolution together with the impact parameter resolution results in proper time resolution better then $40 \mathrm{fs}$ which should allow $\mathrm{LHCb}$ to make a $5 \sigma$ measurement of the $B_{s}^{0}$ mixing parameter $\Delta m_{s}$ up to $68 p s^{-16}$. 

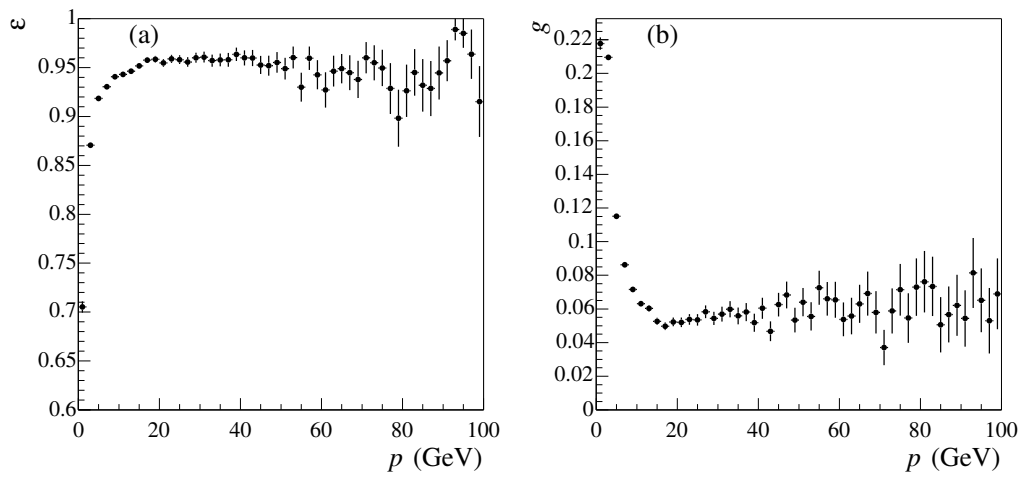

Figure 6. Tracking efficiency (a) and ghost rate (b) for long tracks as a function of momentum, $p$.

\section{Conclusion}

The general $\mathrm{LHCb}$ tracking performance in terms of efficiency, ghost rate, momentum resolution $(\Delta p / p=0.38 \%)$ and vertex resolution leads to a typical resolution on the $B_{s}^{0}$-meson mass of $14 \mathrm{MeV} / \mathrm{c}^{2}$ and to a proper time resolution of $40 \mathrm{fs}$. This proper time resolution is sufficient to observe $\Delta m_{s}$ up to $68 \mathrm{ps}^{-1}$.

The construction of the different sub-detectors is in steady progress and the full physics program will start when LHC will become operational in 2007 .

\section{References}

1. The LHCb Collaboration, P.R.Barbosa et al., LHCb VELO Technical Design Report, CERN-LHCC/2001-011, May 2001.

2. The LHCb Collaboration, Nobrega R. et al., LHCb Reoptimized Detector Design and Performance Technical Design Report, CERN-LHCC/2003-030, Sept. 2003.

3. The LHCb Collaboration, P.R.Barbosa et al.,LHCb Inner Tracker Technical Design Report, CERN-LHCC/2002-029, Nov. 2002.

4. The LHCb Collaboration, P.R.Barbosa et al.,Outer Tracker Technical Design Report, CERN-LHCC/2001-024, Sept. 2001.

5. G.v.Apeldoorn et al.,Beam Tests of Final Modules and Electronics of the LHCb Outer Tracker in 2005, LHCb Note 2005-076, Oct. 2005.

6. O. Leroy, in these proceedings (2006). 